\title{
A COUNTER-EXAMPLE TO THE EQUIVARIANCE STRUCTURE ON SEMI-UNIVERSAL DEFORMATION
}

\author{
AN KHUONG DOAN
}

\begin{abstract}
If $X$ is a projective variety and $G$ is an algebraic group acting algebraically on $X$, we provide a counter-example to the existence of a $G$-equivariant extension on the formal semi-universal deformation of $X$.
\end{abstract}

\section{Contents}

Introduction 1

1. Formal schemes and formal deformations 2

2. The second Hirzebruch surface and its automorphism group 5

3. A formal semi-universal deformation of $\mathbb{F}_{2}$ and formal vector fields on it 7

3.1. Construction of the semi-universal deformation of $\mathbb{F}_{2} \quad 7$

3.2. Formal vector fields on the formal semi-universal deformation of $\mathbb{F}_{2}$

4. The non-existence of $G$-equivariant structure on the formal semi-universal deformation 11

References $\quad 13$

\section{INTRODUCTION}

Let $X$ be an algebraic variety defined over a field $k$ of characteristic zero. Due to Schlessinger's work in [5], the existence of a formal semi-universal deformation (unique up to non-canonical isomorphism), which contains all the information of small deformations of $X$, is assured provided that $H^{1}\left(X, \mathcal{T}_{X}\right)$ and $H^{2}\left(X, \mathcal{T}_{X}\right)$ are finite dimensional vector spaces. These conditions realise for example, if $X$ is a complete scheme over $k$ or an affine scheme with at most isolated singularities (see [6, Corollary 2.4.2]). Now, we equipe $X$ with an action of an algebraic group $G$ defined over $k$. One question arising naturally is whether there exists a formal semi-universal deformation $\pi$ : $\mathcal{X} \rightarrow S$ of $X$, on which we can provide a $G$-action extending the given one on $X$. The answer is positive in the case that $G$ satisfies some vanishing conditions on its cohomology groups, i.e. $H^{1}(G,-)=0$ and $H^{2}(G,-)=0$ for a class of $G$-modules determined by $X$. In particular, these vanishing conditions hold for linearly reductive groups (see [4]). However, we do not know if there exists a non-reductive group whose action on $X$ does not extend to the formal semi-universal deformation of $X$. Therefore, we wish to give an example which illustrates this phenomenon. More

Date: January 22, 2019.

2010 Mathematics Subject Classification. 14D15, 14B10, 13D10.

Key words and phrases. Deformation theory, Moduli theory, Equivariance structure. 
precisely, we prove that the action of the automorphism group of the second Hirzebruch surface $\mathbb{F}_{2}$ does not extend to its formal semi-universal deformation.

Our proof goes as follows. First, we find a nice presentation of $G:=\operatorname{Aut}\left(\mathbb{F}_{2}\right)$. Then we construct a formal semi-universal deformation $\widehat{\mathcal{X}}$ of $\mathbb{F}_{2}$. It turns out that $G$ is non-reductive and that the Lie algebra of $G$ is a 7-dimensional vector space. As a matter of fact, we obtain seven vector fields on $\mathbb{F}_{2}$ with Lie bracket relations induced by those in $\operatorname{Lie}(G)$. Next, we describe the general form of formal vector fields on $\widehat{\mathcal{X}}$. Finally, we conclude the paper by means of contradiction. Suppose that the $G$-action on $\mathbb{F}_{2}$ does extend to a $G$-action on $\widehat{\mathcal{X}}$ then we also have seven formal vector fields on $\widehat{\mathcal{X}}$ whose restrictions on the central fiber are nothing but our initial ones on $\mathbb{F}_{2}$. By manipulating these vector fields with a filtration $F$ given by the vanishing order at 0 , we obtain the existence of a 3-dimensional abelian Lie subalgebra in $\mathfrak{s l}_{2}(K) \times \mathfrak{s l}_{2}(K)$, where $\mathfrak{s l}_{2}(K)$ is the special linear group and $K$ is the field of formal Laurent power series $\mathbb{C}\left[\left[t, t^{-1}\right]\right]$, which is not the case. A remark is in order. Since the semi-universal family of $\mathbb{F}_{2}$ is in fact not universal, another possible way to obtain a contradiction is to use Wavrik's criterion (see [7, Theorem 4.1]) but the calculations are rather complicated.

Acknowledgements. I would like to thank Prof. Bernd Siebert for many useful discussions. Actually, I learned the idea of using the extension of vector fields and their relations as obstructions to the extension of the group action from an unpublished paper of his. This provides a strategy to attack the problem. I am specially thankful to Prof. Julien Grivaux for his careful reading and his comments which help to improve the manuscript. Finally, I am warmly grateful to the referee whose work led to a remarkable improvement of the paper.

\section{Formal SChemes AND FORMAL DEFORMATions}

In this section, by $k$, we always mean a field of characteristic zero. We begin by recalling the definition of formal schemes. For more details, the readers are referred to [2, Chapter III. 9].

Definition 1.1. Let $X$ be a noetherian scheme and let $Y$ be a closed subscheme defined by a sheaf of ideals $\mathcal{J}$. Then we define the formal completion of $X$ along $Y$, denoted $\left(\widehat{X}, \mathcal{O}_{\widehat{X}}\right)$ (sometimes just $\widehat{X})$, to be the following ringed space. We take the topological space $Y$, and on it the sheaf of rings $\mathcal{O}_{\widehat{X}}=\lim _{\longleftarrow} \mathcal{O}_{X} / \mathcal{I}^{n}$. Here we consider each $\mathcal{O}_{X} / \mathcal{I}^{n}$ as sheaf of rings on $Y$

Remark 1.1. For each $n$, let $X_{n}=\left(X, \mathcal{O}_{X} / \mathcal{I}^{n}\right)$. Then we obtain a sequence of closed immersions of schemes

$$
X_{1} \rightarrow X_{2} \rightarrow \cdots \rightarrow X_{n} \rightarrow \cdots .
$$

This expression is helpful in the sequel.

Definition 1.2. A noetherian formal scheme is a locally ringed space $\left(\mathfrak{X}, \mathcal{O}_{\mathfrak{X}}\right)$ which has a finite open cover $\left\{\mathfrak{U}_{i}\right\}$ such that for each $i$, the pair $\left(\mathfrak{U}_{i},\left.\mathcal{O}_{\mathfrak{X}}\right|_{\mathfrak{U}_{i}}\right)$ is isomorphic, as a locally ringed space, to the completion of some noetherian scheme $X_{i}$ along a closed subscheme $Y_{i}$. A morphism of noetherian formal schemes is a morphism as locally ringed spaces.

Example 1.1. If $X$ is any noetherian scheme, and $Y$ is a closed subscheme then the formal completion $\widehat{X}$ of $X$ along $Y$ is a formal scheme.

Example 1.2. For $X=\mathbb{C}^{1}=\operatorname{Spec}(\mathbb{C}[t])$ and $Y=\{0\}$, the formal scheme $\widehat{X}$ is the locally ringed space $\left(Y, \mathcal{O}_{\widehat{X}}\right)$, where the structure sheaf $\mathcal{O}_{\widehat{X}}$ is $\mathbb{C}[[t]]$. We denote $\operatorname{Specf}(\mathbb{C}[[t]]):=\left(Y, \mathcal{O}_{\widehat{X}}\right)$. 
Let $\left(\mathfrak{X}, \mathcal{O}_{\mathfrak{X}}\right)$ be a noetherian formal scheme. We would like to define formal vector fields on $\mathfrak{X}$. Let $\left\{\mathfrak{U}_{i}\right\}$ be a finite open cover of $\mathfrak{X}$ such that for each $i$, the pair $\left(\mathfrak{U}_{i}, \mathcal{O}_{\mathfrak{X}} \mid \mathfrak{U}_{i}\right)$ is the formal completion $\left(\widehat{X}_{i}, \mathcal{O}_{\widehat{X}_{i}}\right)$ of some noetherian scheme $X_{i}$ along a closed subscheme $Y_{i}$. By Remark 1.1, for each $i$ we have a sequence of closed immersions of schemes

$$
X_{i 1} \rightarrow X_{i 2} \rightarrow \cdots \rightarrow X_{i n} \rightarrow \cdots
$$

Definition 1.3. A formal vector field on a noetherian formal scheme $\mathfrak{X}$ is a sequence of vector fields $\left\{v_{i, n}\right\}$ such that

(i) Each $v_{i, n}$ is a usual vector field on the scheme $X_{i, n}$,

(ii) $v_{i, n}$ induces $v_{i, n-1}$ via the natural inclusion $X_{i, n-1} \rightarrow X_{i, n}$,

(iii) $\left.v_{i, n}\right|_{X_{i, n} \cap X_{j, n}}=\left.v_{j, n}\right|_{X_{j, n} \cap X_{i, n}}$.

Next, we turn to the notion of infinitesimal deformations and that of formal deformations. Let $X$ be an algebraic scheme and let $A$ be an artinian local $k$-algebra with residue field $k$. An infinitesimal deformation of $X$ is a deformation of $X$ over the $\operatorname{scheme} \operatorname{Spec}(A)$, i.e. a commutative diagram

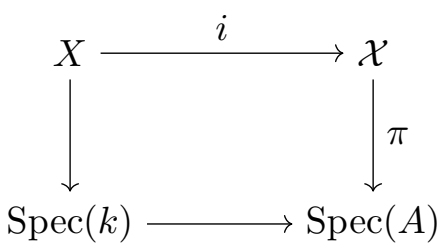

where $\pi: \mathcal{X} \rightarrow \operatorname{Spec}(A)$ is a flat surjective morphism of schemes.

Now, let $A$ be a complete local noetherian $k$-algebra with the unique maximal ideal $\mathfrak{m}$ and with residue $k$.

Definition 1.4. A formal deformation of $X$ over $A$ is a sequence $\left\{\nu_{n}\right\}$ of infinitesimal deformations of $X$, in which $\nu_{n}$ is represented by a deformation

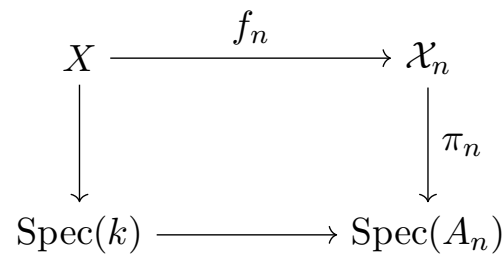

where $A_{n}=A / \mathfrak{m}^{n+1}$, such that for all $n \geq 1, \nu_{n}$ induces $\nu_{n-1}$ by pullback under the natural inclusion $\operatorname{Spec}\left(A_{n-1}\right) \rightarrow \operatorname{Spec}\left(A_{n}\right)$, i.e. $\nu_{n-1}$ is also represented by the deformation

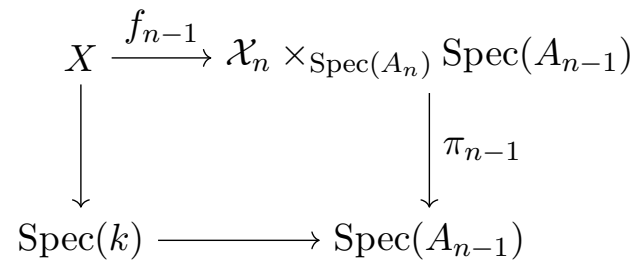


In the language of formal schemes, we can write $\left\{\nu_{n}\right\}$ as the morphism of formal schemes

$$
\widehat{\pi}: \widehat{\mathcal{X}} \rightarrow \operatorname{Specf}(A)
$$

where

$$
\widehat{\mathcal{X}}=\left(X, \lim _{\leftarrow} \mathcal{O}_{\mathcal{X}_{n}}\right) \text { and } \widehat{\pi}=\lim _{\leftarrow} \pi_{n} .
$$

Here, $\mathcal{O}_{\mathcal{X}_{n}}$ is the structure sheaf on $\mathcal{X}_{n}$ and $\operatorname{Specf}(A)$ is the formal scheme obtained by completing $\operatorname{Spec}(A)$ along its closed point, which corresponds to the unique maximal ideal of $A$. The easiest way to construct formal deformations is to build out of usual ones. This leads to the definition of formal deformation associated to a given deformation. Let $X$ be a projective scheme and let $\nu$ be a deformation represented by

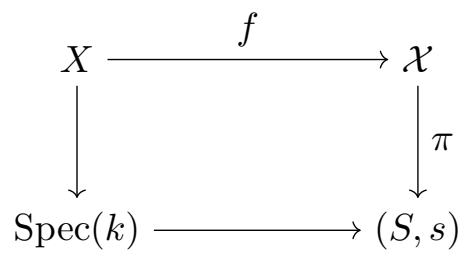

where $S=\operatorname{Spec}(B)$ for some $k$-algebra of finite type $B$ and $s$ is a $k$-rational point of $S$.

Definition 1.5. The formal deformation associated to $\nu$ is defined to be the sequence of deformations $\left\{\nu_{n}\right\}$ where each $\nu_{n}$ is the pullback of $\nu$ under the natural closed embedding

$$
S_{n}:=\operatorname{Spec}\left(\mathcal{O}_{S, s} / \mathfrak{m}_{s}^{n+1}\right) \rightarrow S
$$

where $\mathfrak{m}_{s}$ is the unique maximal ideal of the local ring $\mathcal{O}_{S, s}$.

Remark 1.2. Note that $\left\{\nu_{n}\right\}$ is formal because of the isomorphism

$$
\mathcal{O}_{S, s} / \mathfrak{m}_{s}^{n+1} \cong \widehat{\mathcal{O}}_{S, s} / \widehat{\mathfrak{m}}_{s}^{n+1}
$$

for all $n$.

To end this section, we introduce a very interesting kind of (formal) deformations, namely, the kind of $G$-equivariant (formal) ones, which is of central interest of the article. Let $G$ be a $k$-algebraic group acting algebraically on a projective variety $X$ and $A$ an artinian local $k$-algebra.

Definition 1.6. A G-equivariant infinitesimal deformation of $X$ over $\operatorname{Spec}(A)$ is a usual deformation of $X$, i.e. a commutative diagram

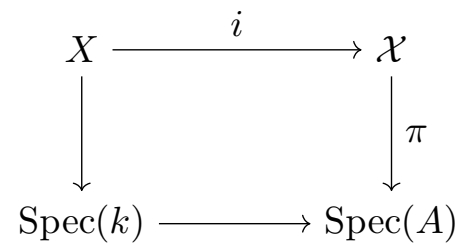

where $\mathcal{X}$ and $\operatorname{Spec}(A)$ are equipped with $G$-actions in a way that any map appearing in the above diagram is $G$-equivariant. In particular, the restriction of the $G$-action on $\mathcal{X}$ on the central fiber is nothing but the initial $G$-action on $X$. 
Finally, we give the definition of $G$-equivariant formal deformations and then we show how to produce formal vector fields from $G$-equivariant formal deformations.

Definition 1.7. A G-equivariant formal deformation of $X$ over a complete local noetherian $k$ algebra $A$ with the unique maximal ideal $\mathfrak{m}$ is a formal deformation of $X$, i.e. a sequence $\left\{\nu_{n}\right\}$ of infinitestimal deformations of $X$, in which $\nu_{n}$ is represented by a $G$-infinitesimal deformation

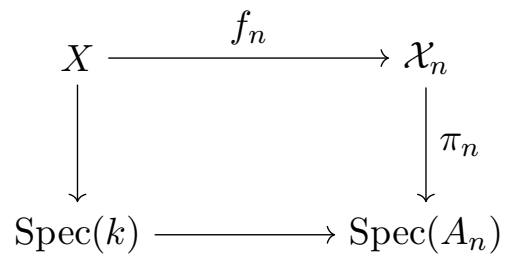

where $A_{n}=A / \mathfrak{m}^{n+1}$, such that for all $n \geq 1$, the $G$-equivariant deformation $\nu_{n}$ induces the $G$ equivariant deformation $\nu_{n-1}$ by pullback under the natural inclusion $\operatorname{Spec}\left(A_{n-1}\right) \rightarrow \operatorname{Spec}\left(A_{n}\right)$.

As before, we can write $\left\{\nu_{n}\right\}$ as the $G$-equivariant morphism of formal schemes

$$
\widehat{\pi}: \widehat{\mathcal{X}} \rightarrow \operatorname{Specf}(A)
$$

where

$$
\widehat{\mathcal{X}}=\left(X, \lim _{\leftarrow} \mathcal{O}_{\mathcal{X}_{n}}\right) \text { and } \widehat{\pi}=\lim _{\leftarrow} \pi_{n} .
$$

Here, the $G$-equivariance of $\widehat{\pi}$ means that $\widehat{\pi}$ is an inverse limit of $G$-equivariant morphisms of schemes $\pi_{n}$. On one hand, on each $n^{\text {th }}$-infinitesimal neighborhood, $G$-actions on $\mathcal{X}_{n}$ and on $\operatorname{Spec}\left(A_{n}\right)$ induce vector fields on $\mathcal{X}_{n}$ and on $\operatorname{Spec}\left(A_{n}\right)$, respectively. They are related by the fact that the differential of $\pi_{n}$ always maps the former ones to the latter ones. On the other hand, these induced vector fields on $\mathcal{X}_{n}$ and on $\operatorname{Spec}\left(A_{n}\right)$ are also induced by those on $\mathcal{X}_{n+1}$ and on $\operatorname{Spec}\left(A_{n+1}\right)$, respectively, via the natural inclusion $\operatorname{Spec}\left(A_{n}\right) \rightarrow \operatorname{Spec}\left(A_{n+1}\right)$. Therefore, we obtain formal vector fields, induced by the $G$-actions, on $\widehat{\mathcal{X}}$ and on $\operatorname{Specf}(A)$, respectively.

\section{The SECOND Hirzebruch SURFACE AND its AUtomorPhism Group}

For the rest of the paper, we assume that $k$ is the field of complex numbers $\mathbb{C}$. The geneneral linear group $\mathrm{GL}(2, \mathbb{C})$ has an obvious linear action on $\mathbb{C}^{2}$. This induces an action on the $\mathbb{C}$-vector space of polynomials in two variables $\mathbb{C}[X, Y]$. Since the subspace of homogeneous polynomials of degree 2 , denoted by $\mathbb{C}[X, Y]_{2}$, is $\mathrm{GL}(2, \mathbb{C})$-invariant then we have a $\mathrm{GL}(2, \mathbb{C})$-action on $\mathbb{C}[X, Y]_{2}$. More precisely, for $g=\left(\begin{array}{ll}a & b \\ c & d\end{array}\right) \in \mathrm{GL}(2, \mathbb{C})$ and $f=a_{0} X^{2}+a_{1} X Y+a_{2} Y^{2} \in \mathbb{C}[X, Y]_{2}$, the action of $g$ on $f$ is given by the linear substitution

$$
\left(\begin{array}{l}
X \\
Y
\end{array}\right):=\left(\begin{array}{ll}
a & b \\
c & d
\end{array}\right)\left(\begin{array}{l}
X \\
Y
\end{array}\right)
$$

i.e.

$$
\begin{aligned}
g . f & =a_{0}(a X+b Y)^{2}+a_{1}(a X+b Y)(c X+d Y)+a_{2}(c X+d Y)^{2} \\
& =\left(a^{2} a_{0}+a c a_{1}+c^{2} a_{2}\right) X^{2}+\left(2 a b a_{0}+(a d+b c) a_{1}+2 c d a_{2}\right) X Y+\left(b^{2} a_{0}+b d a_{1}+d^{2} a_{2}\right) Y^{2} .
\end{aligned}
$$


Identifying $\mathbb{C}[X, Y]_{2}$ with $\mathbb{C}^{3}$, the corresponding action on $\mathbb{C}^{3}$ can be written as

$$
g \cdot\left(a_{0}, a_{1}, a_{2}\right)=\left(\begin{array}{ccc}
a^{2} & a c & c^{2} \\
2 a b & a d+b c & 2 c d \\
b^{2} & b d & d^{2}
\end{array}\right)\left(\begin{array}{l}
a_{0} \\
a_{1} \\
a_{2}
\end{array}\right) .
$$

This action gives rise to an algebraic group $H$ which is the semi-product of $\mathbb{C}^{3}$ and $\operatorname{GL}(2, \mathbb{C})$, i.e.

$$
H:=\mathbb{C}^{3} \rtimes \mathrm{GL}(2, \mathbb{C}) .
$$

This is a non-reductive linear group. Recall that an algebraic group $K$ is reductive if the greatest connected normal subgroup $R_{u}(K)$ of $K$ is trivial. In our case, $R_{u}(H)=\mathbb{C}^{3}$.

Next, we recall the definition of the second Hirzebruch surface. Let $\mathbb{P}\left(\mathcal{O}_{\mathbb{P}^{1}}(2) \oplus \mathcal{O}_{\mathbb{P}^{1}}\right)$ be the projectivization of $\mathcal{O}_{\mathbb{P}^{1}}(2) \oplus \mathcal{O}_{\mathbb{P}^{1}}$, where $\mathcal{O}_{\mathbb{P}^{1}}$ is the structure sheaf of the projective space $\mathbb{P}^{1}$.

Definition 2.1. The second Hirzebruch surface is defined to be $\mathbb{P}\left(\mathcal{O}_{\mathbb{P}^{1}}(2) \oplus \mathcal{O}_{\mathbb{P}^{1}}\right)$.

Proposition 2.1. The second Hirzebruch surface is isomorphic to the variety

$$
\mathbb{F}_{2}:=\left\{([x: y: z],[u: v]) \in \mathbb{P}^{2} \times \mathbb{P}^{1} \mid y v^{2}=z u^{2}\right\} .
$$

Proof. Let $\sigma: \mathbb{P}\left(\mathcal{O}_{\mathbb{P}^{1}}(2) \oplus \mathcal{O}_{\mathbb{P}^{1}}\right) \rightarrow \mathbb{P}^{1}$ be the canonical projection of the projectivization $\mathbb{P}\left(\mathcal{O}_{\mathbb{P}^{1}}(2) \oplus\right.$ $\left.\mathcal{O}_{\mathbb{P}^{1}}\right)$, let $U=\operatorname{Spec}(\mathbb{C}[v])$ and $U^{\prime}=\operatorname{Spec}\left(\mathbb{C}\left[v^{\prime}\right]\right)$ such that $v^{\prime} v=1$ on $U \cap U^{\prime}$. Then $\mathbb{P}\left(\mathcal{O}_{\mathbb{P}^{1}}(2) \oplus \mathcal{O}_{\mathbb{P}^{1}}\right)$ has the following presentation

$$
\mathbb{P}\left(\mathcal{O}_{\mathbb{P}^{1}}(2) \oplus \mathcal{O}_{\mathbb{P}^{1}}\right)=\sigma^{-1}(U) \cup \sigma^{-1}\left(U^{\prime}\right)=\left(U \times \mathbb{P}^{1}\right) \cup\left(U^{\prime} \times \mathbb{P}^{1}\right)
$$

such that on the intersection of the affine open sets $V=\operatorname{Spec}(\mathbb{C}[v, y]) \subset U \times \mathbb{P}^{1}$ and $V^{\prime}=$ $\operatorname{Spec}\left(\mathbb{C}\left[v^{\prime}, y^{\prime}\right]\right) \subset U \times \mathbb{P}^{1}$, we have

$$
\left\{\begin{array}{l}
v v^{\prime}=1 \\
y^{\prime}=y v^{2}
\end{array}\right.
$$

So, an open covering of $\mathbb{F}_{2}$ is given by the open embeddings

$$
\begin{aligned}
\rho_{1}: U \times \mathbb{P}^{1} & \rightarrow \mathbb{F}_{2} \\
(v,[x: y]) & \mapsto\left(\left[x: y: y v^{2}\right],[1: v]\right)
\end{aligned}
$$

and

$$
\begin{aligned}
\rho_{2}: U^{\prime} \times \mathbb{P}^{1} & \rightarrow \mathbb{F}_{2} \\
\left(v^{\prime},\left[x^{\prime}: y^{\prime}\right]\right) & \mapsto\left(\left[x^{\prime}: y^{\prime} v^{\prime 2}: y^{\prime}\right],\left[v^{\prime}: 1\right]\right),
\end{aligned}
$$

which glue to give an isomorphism $\rho: \mathbb{P}\left(\mathcal{O}_{\mathbb{P}^{1}}(2) \oplus \mathcal{O}_{\mathbb{P}^{1}}\right) \rightarrow \mathbb{F}_{2}$.

Now, the algebraic group $H$ acts on the second Hirzebruch surface

$$
\mathbb{F}_{2}=\left\{([x: y: z],[u: v]) \in \mathbb{P}^{2} \times \mathbb{P}^{1} \mid y v^{2}=z u^{2}\right\}
$$

in the following manner: for $p=([x: y: z],[u: v]) \in \mathbb{F}_{2}$ and $g=\left(\left(a_{0}, a_{1}, a_{2}\right)^{t},\left(\begin{array}{ll}a & b \\ c & d\end{array}\right)\right) \in H$,

$g \cdot p=\left\{\begin{array}{ll}\left(\left[x u^{2}+y\left(a_{0} v^{2}+a_{1} u v+a_{2} u^{2}\right): y(a u+b v)^{2}: y(c u+d v)^{2}\right],[a u+b v: c u+d v]\right) & \text { if } u \neq 0 \\ \left(\left[x v^{2}+z\left(a_{0} v^{2}+a_{1} u v+a_{2} u^{2}\right): z(a u+b v)^{2}: z(c u+d v)^{2}\right],[a u+b v: c u+d v]\right) & \text { if } v \neq 0\end{array}\right.$.

The following theorem is well-known (see [1, Section 6.1]). 
Theorem 2.1. The group of automorphisms of $\mathbb{F}_{2}$ is exactly the quotient of $H$ by the subgroup $I$ consisting of diagonal matrices of the form $\left(\begin{array}{ll}\mu & 0 \\ 0 & \mu\end{array}\right)$ where $\mu \in \mathbb{C}$ such that $\mu^{2}=1$.

\section{A formal SEMI-UNIVERSAL DEFORMATION OF $\mathbb{F}_{2}$ AND FORMAL VECTOR FIELDS ON IT}

3.1. Construction of the semi-universal deformation of $\mathbb{F}_{2}$. We shall follow the construction given in [4, Example 1.2.2.(iii)]. Consider two copies of $\mathbb{C} \times \mathbb{C} \times \mathbb{P}^{1}$ given by $W:=\operatorname{Proj}(\mathbb{C}[t, v, x, y])$ and $W^{\prime}:=\operatorname{Proj}\left(\mathbb{C}\left[t^{\prime}, v^{\prime}, x^{\prime}, y^{\prime}\right]\right)$ (note that these two rings are graded with respect to $x, y$ and $x^{\prime}, y^{\prime}$, respectively). Consider the affine subsets $\operatorname{Spec}(\mathbb{C}[t, v, y]) \subset W, \operatorname{Spec}\left(\mathbb{C}\left[t^{\prime}, v^{\prime}, y^{\prime}\right]\right) \subset W^{\prime}$ and then glue them along the open subsets

$$
\operatorname{Spec}\left(\mathbb{C}\left[t, v, v^{-1}, y\right]\right) \subset \operatorname{Spec}(\mathbb{C}[t, v, y])
$$

and

$$
\operatorname{Spec}\left(\mathbb{C}\left[t^{\prime}, v^{\prime}, v^{\prime-1}, y^{\prime}\right]\right) \subset \operatorname{Spec}\left(\mathbb{C}\left[t^{\prime}, v^{\prime}, y^{\prime}\right]\right)
$$

by the rules

$$
\left\{\begin{array}{l}
v v^{\prime}=1 \\
x^{\prime}=x \\
y^{\prime}=y v^{2}-t v x \\
t^{\prime}=t
\end{array}\right.
$$

This gives a gluing of $W$ and $W^{\prime}$ along

$$
\operatorname{Proj}\left(\mathbb{C}\left[t, v, v^{-1}, x, y\right]\right) \text { and } \operatorname{Proj}\left(\mathbb{C}\left[t^{\prime}, v^{\prime}, v^{\prime-1}, x^{\prime}, y^{\prime}\right]\right) \text {. }
$$

We denote the resulting scheme by $\mathcal{W}$. In other words, if we let $(t, v,[x: y])$ and $\left(t^{\prime}, v^{\prime},\left[x^{\prime}: y^{\prime}\right]\right)$ be the coordinates on $W=\mathbb{C} \times \mathbb{C} \times \mathbb{P}^{1}$ and on $W^{\prime}=\mathbb{C} \times \mathbb{C} \times \mathbb{P}^{1}$, respectively. Then $W$ is obtained by glue $W$ and $W^{\prime}$ according to the rules (3.1). Now, let $\pi: \mathcal{W} \rightarrow \mathbb{C}$ be the morphism induced by the projections.

Theorem 3.1. The familly $\pi: \mathcal{W} \rightarrow \mathbb{C}=\operatorname{Spec}(\mathbb{C}[t])$ is a semi-universal deformation of $\mathbb{F}_{2}$. Moreover,

$$
\pi^{-1}(t)=\left\{\begin{array}{l}
\mathbb{F}_{2} \text { if } t=0 \\
\mathbb{P}^{1} \times \mathbb{P}^{1} \text { otherwise }
\end{array}\right.
$$

Proof. The map $\pi$ is obviously surjective by construction. Since $\pi$ is locally a projection, it is a flat morphism. Moreover, by Proposition 2.1, $\mathcal{W}_{0}=\pi^{-1}(0)=\mathbb{F}_{2}$. Then $\pi: \mathcal{W} \rightarrow \mathbb{C}$ is a deformation of $\mathbb{F}_{2}$. Next, let $\mathcal{W}^{*}=\pi^{-1}\left(\mathbb{C}^{*}\right)$ and $\pi^{*}: \mathcal{W}^{*} \rightarrow \mathbb{C}^{*}$ is the restriction of $\pi$ on $\mathbb{C}^{*}=\operatorname{Spec}\left(\mathbb{C}\left[t, t^{-1}\right]\right)$. We shall prove that $\mathcal{W}^{*}$ is in fact isomorphic to $\mathbb{C}^{*} \times \mathbb{P}^{1} \times \mathbb{P}^{1}$. Indeed, consider the following open embeddings

$$
\begin{aligned}
\phi: \mathbb{C}^{*} \times \mathbb{C} \times \mathbb{P}^{1} & \rightarrow \mathbb{C}^{*} \times \mathbb{P}^{1} \times \mathbb{P}^{1} \\
(t, v,[x: y]) & \mapsto(t,[1: v],[t y: v y-t x])
\end{aligned}
$$

and 


$$
\begin{aligned}
\phi^{\prime}: \mathbb{C}^{*} \times \mathbb{C} \times \mathbb{P}^{1} & \rightarrow \mathbb{C}^{*} \times \mathbb{P}^{1} \times \mathbb{P}^{1} \\
\left(t^{\prime}, v^{\prime},\left[x^{\prime}: y^{\prime}\right]\right) & \mapsto\left(t^{\prime},\left[v^{\prime}: 1\right],\left[t^{\prime} v^{\prime} y^{\prime}+t^{\prime 2} x^{\prime}: y^{\prime}\right]\right) .
\end{aligned}
$$

By the gluing condition (3.1), we have that

$$
\begin{aligned}
\left(t^{\prime},\left[v^{\prime}: 1\right],\left[t^{\prime} v^{\prime} y^{\prime}+t^{\prime 2} x^{\prime}: y^{\prime}\right]\right) & =\left(t^{\prime},\left[v^{\prime}: 1\right],\left[t^{\prime} v^{\prime}\left(y v^{2}-t v x\right)+t^{\prime 2} x^{\prime}: y v^{2}-t v x\right]\right) \\
& =\left(t^{\prime},\left[v^{\prime}: 1\right],\left[t^{\prime} y v: y v^{2}-t v x\right]\right) \\
& =(t,[1: v],[t y: y v-t x]) .
\end{aligned}
$$

Hence, the above two morphisms glue to give an isomorphism

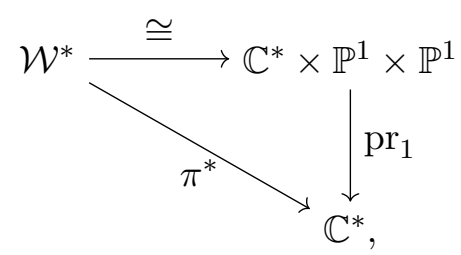

which means precisely that $\pi^{*}: \mathcal{W}^{*} \rightarrow \mathbb{C}^{*}$ is the trivial family whose fibers are all isomorphic to $\mathbb{P}_{1} \times \mathbb{P}_{1}$. In particular, for $t \in \mathbb{C}^{*}, \pi^{-1}(t)=\left(\pi^{*}\right)^{-1}(t)=\mathbb{P}_{1} \times \mathbb{P}_{1}$.

It remains to prove that the family $\pi: \mathcal{W} \rightarrow \mathbb{C}$ is actually semi-universal. One way to see it is to compute the Kodaira-Spencer map $\mathcal{K}_{\pi, 0}$ of $\pi$ at 0 . This map is uniquely determined by the element $\mathcal{K}_{\pi, 0}\left(\frac{d}{d t}\right)$ in $H^{1}\left(\mathbb{F}_{2}, \mathcal{T}_{\mathbb{F}_{2}}\right)$. By definition, $\mathcal{K}_{\pi, 0}\left(\frac{d}{d t}\right)$ represents the first order deformation of $\mathbb{F}_{2}$, obtained by gluing $W_{0}:=\operatorname{Proj}(\mathbb{C}[\epsilon, v, x, y])$ and $W_{0}^{\prime}:=\operatorname{Proj}\left(\mathbb{C}\left[\epsilon, v^{\prime}, x^{\prime}, y^{\prime}\right]\right)$ along $\operatorname{Proj}\left(\mathbb{C}\left[\epsilon, v, v^{-1}, x, y\right]\right)$ and $\operatorname{Proj}\left(\mathbb{C}\left[\epsilon, v^{\prime}, v^{\prime-1}, x^{\prime}, y^{\prime}\right]\right)$ by the rules

$$
\left\{\begin{array}{l}
v v^{\prime}=1 \\
y^{\prime}=y v^{2}-\epsilon v
\end{array},\right.
$$

where $\mathbb{C}[\epsilon]$ is the ring of complex dual numbers. Hence, $\mathcal{K}_{\pi, 0}\left(\frac{d}{d t}\right) \in H^{1}\left(\mathcal{U}, \mathcal{T}_{\mathbb{F}_{2}}\right)$ is the 1-cocycle which corresponds to the vector field $\left\{-v \frac{\partial}{\partial y}\right\}$ on $W_{0} \cap W_{0}^{\prime}$, where $\mathcal{U}$ is the covering $\left\{W_{0}, W_{0}^{\prime}\right\}$. By [3, Example B.11(iii)], we see that $\left\{-v \frac{\partial}{\partial y}\right\}$ is nonzero and $\operatorname{dim}_{\mathbb{C}} H^{1}\left(\mathbb{F}_{2}, \mathcal{T}_{\mathbb{F}_{2}}\right)=1$. Thus, the Kodaira-Spencer map is an isomorphism and so $\pi: \mathcal{W} \rightarrow \mathbb{C}$ is semi-universal.

Another useful presentation of $\mathcal{W}$ is given as follows.

Proposition 3.1. The scheme $\mathcal{W}$ is isomorphic to the surface

$$
\mathcal{X}:=\left\{([x: y: z],[u: v], t) \in \mathbb{P}^{2} \times \mathbb{P}^{1} \times \mathbb{C} \mid y v^{2}-z u^{2}-t x u v=0\right\} .
$$

Proof. We have an open covering of $\mathcal{X}$ given by the open embeddings

$$
\begin{aligned}
\rho_{1}: \mathbb{C} \times \mathbb{C} \times \mathbb{P}^{1} & \rightarrow \mathcal{X} \\
(t, v,[x: y]) & \mapsto\left(\left[x: y: y v^{2}-t v\right],[1: v], t\right)
\end{aligned}
$$


and

$$
\begin{aligned}
\rho_{2}: \mathbb{C} \times \mathbb{C} \times \mathbb{P}^{1} & \rightarrow \mathcal{X} \\
\left(t^{\prime}, v^{\prime},\left[x^{\prime}: y^{\prime}\right]\right) & \mapsto\left(\left[x^{\prime}: y^{\prime} v^{\prime 2}+t^{\prime} v^{\prime}: y^{\prime}\right],\left[v^{\prime}: 1\right], t\right)
\end{aligned}
$$

which glue to give an isomorphism $\mathcal{W} \cong \mathcal{X}$.

Remark 3.1. By Proposition 2.1 and by Proposition 3.1, from now on, we use interchangeably between $\mathbb{F}_{2}, \mathcal{X}$ and $\mathbb{P}\left(\mathcal{O}_{\mathbb{P}^{1}}(2) \oplus \mathcal{O}_{\mathbb{P}^{1}}\right), \mathcal{W}$, respectively.

3.2. Formal vector fields on the formal semi-universal deformation of $\mathbb{F}_{2}$. The formal deformation associated to $\mathcal{W}, \widehat{\pi}: \widehat{\mathcal{W}} \rightarrow \operatorname{Specf}(\mathbb{C}[[t]])$ is a formal semi-universal deformation of $\mathbb{F}_{2}$ (here $\mathbb{C}[[t]]$ is the ring of formal power series in the variable $t$ ). We will give explicit descriptions of formal vector fields on $\widehat{\mathcal{W}}$. Consider the covering $\left\{W, W^{\prime}\right\}$ where $W:=\operatorname{Proj}(\mathbb{C}[t, v, x, y])$ and $W^{\prime}:=\operatorname{Proj}\left(\mathbb{C}\left[t^{\prime}, v^{\prime}, x^{\prime}, y^{\prime}\right]\right)$, as before. A formal vector field on $W$ is of the form

$$
g_{1}(v, t) \frac{\partial}{\partial v}+\left(\alpha_{1}(v, t) y^{2}+\beta_{1}(v, t) y+\gamma_{1}(v, t)\right) \frac{\partial}{\partial y}+k_{1}(t) \frac{\partial}{\partial t}
$$

where $g_{1}, \alpha_{1}, \beta_{1}, \gamma_{1}, k_{1}$ are formal power series in the variable $t$. Likewise, a formal vector field on $W^{\prime}$ is of the form

$$
g_{2}\left(v^{\prime}, t^{\prime}\right) \frac{\partial}{\partial v^{\prime}}+\left(\alpha_{2}\left(v^{\prime}, t^{\prime}\right) y^{\prime 2}+\beta_{2}\left(v^{\prime}, t^{\prime}\right) y^{\prime}+\gamma_{2}\left(v^{\prime}, t^{\prime}\right)\right) \frac{\partial}{\partial y^{\prime}}+k_{2}\left(t^{\prime}\right) \frac{\partial}{\partial t^{\prime}}
$$

where $g_{2}, \alpha_{2}, \beta_{2}, \gamma_{2}, k_{2}$ are formal power series in the variable $t^{\prime}$. Therefore, a vector field on $\mathcal{W}$ which is of the form (3.2) on $W$ and of the form (3.3) on $W^{\prime}$ must satisfy the relation

$$
\begin{aligned}
& g_{1}(v, t) \frac{\partial}{\partial v}+\left(\alpha_{1}(v, t) y^{2}+\beta_{1}(v, t) y+\gamma_{1}(v, t)\right) \frac{\partial}{\partial y}+k_{1}(t) \frac{\partial}{\partial t} \\
& =g_{2}\left(v^{\prime}, t^{\prime}\right) \frac{\partial}{\partial v^{\prime}}+\left(\alpha_{2}\left(v^{\prime}, t^{\prime}\right) y^{\prime 2}+\beta_{2}\left(v^{\prime}, t^{\prime}\right) y^{\prime}+\gamma_{2}\left(v^{\prime}, t^{\prime}\right)\right) \frac{\partial}{\partial y^{\prime}}+k_{2}\left(t^{\prime}\right) \frac{\partial}{\partial t^{\prime}}
\end{aligned}
$$

on the overlapping open set $W \cap W^{\prime}$.

Lemma 3.1. A global formal vector field on $\widehat{\mathcal{W}}$ whose restriction on $W$ is

$$
g_{1}(v, t) \frac{\partial}{\partial v}+\left(\alpha_{1}(v, t) y^{2}+\beta_{1}(v, t) y+\gamma_{1}(v, t)\right) \frac{\partial}{\partial y}+k_{1}(t) \frac{\partial}{\partial t}
$$

must satisfy the following

$$
\left\{\begin{array}{l}
g_{1}(v, t)=A(t) v^{2}+B(t) v+C(t) \\
\alpha_{1}(v, t)=a(t) v^{2}+b(t) v+c(t) \\
\beta_{1}(v, t)=-2[a(t) t+A(t)] v+e(t) \\
\gamma_{1}(v, t)=t^{2} a(t)+t A(t)
\end{array}\right.
$$

where $A, B, C, a, b, c, e, k_{1}$ are formal power series in the variable $t$ with a relation

$$
b(t) t^{2}+e(t) t+B(t) t-k_{1}(t)=0 .
$$


Proof. By (3.1), we have

$$
\left\{\begin{array}{l}
y=v^{\prime 2} y^{\prime}+t v^{\prime} \\
v=\frac{1}{v^{\prime}} \\
t=t^{\prime} \\
\partial_{v}=-v^{\prime 2} \partial_{v^{\prime}}+\left(2 y^{\prime} v^{\prime}+t\right) \partial_{y^{\prime}} \\
\partial_{y}=\frac{1}{v^{\prime 2}} \partial_{y^{\prime}} \\
\partial_{t^{\prime}}=-\frac{1}{v^{\prime}} \partial_{y^{\prime}}+\partial_{t^{\prime}}
\end{array}\right.
$$

Substituting (3.7) into the left hand side of (3.4) and equalizing, we get that

$$
\left\{\begin{array}{l}
g_{2}\left(v^{\prime}, t^{\prime}\right)=-v^{\prime 2} g_{1}\left(\frac{1}{v^{\prime}}, t^{\prime}\right) \\
\alpha_{2}\left(v^{\prime}, t^{\prime}\right)=v^{\prime 2} \alpha_{1}\left(\frac{1}{v^{\prime}}, t^{\prime}\right) \\
\beta_{2}\left(v^{\prime}, t^{\prime}\right)=2 t^{\prime} v^{\prime} \alpha_{1}\left(\frac{1}{v^{\prime}}, t^{\prime}\right)+\beta_{1}\left(\frac{1}{v^{\prime}}, t^{\prime}\right)+2 v^{\prime} g_{1}\left(\frac{1}{v^{\prime}}, t^{\prime}\right) \\
\gamma_{2}\left(v^{\prime}, t^{\prime}\right)=t^{\prime 2} \alpha_{1}\left(\frac{1}{v^{\prime}}, t^{\prime}\right)+\frac{t^{\prime}}{v^{\prime}} \beta_{1}\left(\frac{1}{v^{\prime}}, t^{\prime}\right)+\frac{1}{v^{\prime 2}} \gamma_{1}\left(v^{\prime}, t^{\prime}\right)+t^{\prime} g_{1}\left(\frac{1}{v^{\prime}}, t^{\prime}\right)-\frac{k_{1}\left(t^{\prime}\right)}{v^{\prime}}
\end{array}\right.
$$

which implies that

$$
\left\{\begin{array}{l}
g_{1}(v, t)=A(t) v^{2}+B(t) v+C(t) \\
\alpha_{1}(v, t)=a(t) v^{2}+b(t) v+c(t) \\
\beta_{1}(v, t)=-2[a(t) t+A(t)] v+e(t) \\
\gamma_{1}(v, t)=t^{2} a(t)+t A(t)
\end{array}\right.
$$

where $A, B, C, a, b, c, e$ are formal power series in the variable $t$ with a relation

$$
b(t) t^{2}+e(t) t+B(t) t-k_{1}(t)=0 .
$$

This constraint comes from the coefficient of $\frac{1}{v^{\prime}}$ in the fourth equation in (3.8).

Remark 3.2. If $t=0$ then (3.5) becomes

$$
\left\{\begin{array}{l}
g_{1}(v)=A v^{2}+B v+C \\
\alpha_{1}(v)=a v^{2}+b v+c \\
\beta_{1}(v)=-2 A v+e \\
\gamma_{1}(v, t)=0
\end{array}\right.
$$

which agrees with Kodaira's calculations of vector fields on $\mathcal{W}_{0}=\mathbb{F}_{2}$ (see [3, Page 75]). In particular, we have seven linearly independent vector fields on $\mathbb{F}_{2}$. If $t$ is non-zero and fixed then we have six linearly independent vector fields on the fiber $\mathcal{W}_{t}$, which is due to the existence of the relation (3.6). 


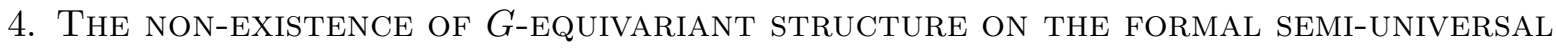 DEFORMATION}

The Lie algebra of $G:=\operatorname{Aut}\left(\mathbb{F}_{2}\right)$ is $\mathbb{C}^{3} \times M(2, \mathbb{C})$, which is evidently 7-dimensional . A $\mathbb{C}$-basis of $\mathrm{Lie}(\mathrm{G})$ is given by the following elements

$$
\left\{\begin{array}{l}
e_{1}=(1,0,0) \times\left(\begin{array}{ll}
0 & 0 \\
0 & 0
\end{array}\right), e_{2}=(0,0,1) \times\left(\begin{array}{ll}
0 & 0 \\
0 & 0
\end{array}\right), e_{3}=(0,0,0) \times\left(\begin{array}{ll}
0 & 0 \\
1 & 0
\end{array}\right), \\
e_{4}=(0,1,0) \times\left(\begin{array}{ll}
0 & 0 \\
0 & 0
\end{array}\right), e_{5}=(0,0,0) \times\left(\begin{array}{ll}
1 & 0 \\
0 & 0
\end{array}\right), e_{6}=(0,0,0) \times\left(\begin{array}{ll}
0 & 0 \\
0 & 1
\end{array}\right), \\
e_{7}=(0,0,0) \times\left(\begin{array}{ll}
0 & 1 \\
0 & 0
\end{array}\right) .
\end{array}\right.
$$

Then the $G$-action gives us 7 vector fields $E_{1}^{\prime}, \ldots, E_{7}^{\prime}$ on $\mathbb{F}_{2}$ with the relations

$$
\begin{aligned}
& \left\{\begin{array}{l}
{\left[E_{1}^{\prime}, E_{2}^{\prime}\right]=0} \\
{\left[E_{1}^{\prime}, E_{3}^{\prime}\right]=-2 E_{4}^{\prime}} \\
{\left[E_{1}^{\prime}, E_{4}^{\prime}\right]=0} \\
{\left[E_{1}^{\prime}, E_{5}^{\prime}\right]=0} \\
{\left[E_{1}^{\prime}, E_{6}^{\prime}\right]=-2 E_{1}^{\prime}} \\
{\left[E_{1}^{\prime}, E_{7}^{\prime}\right]=0,}
\end{array}\right. \\
& \left\{\begin{array} { l } 
{ [ E _ { 4 } ^ { \prime } , E _ { 5 } ^ { \prime } ] = - E _ { 4 } ^ { \prime } } \\
{ [ E _ { 4 } ^ { \prime } , E _ { 6 } ^ { \prime } ] = - E _ { 4 } ^ { \prime } } \\
{ [ E _ { 4 } ^ { \prime } , E _ { 7 } ^ { \prime } ] = - E _ { 1 } ^ { \prime } , }
\end{array} \quad \left\{\begin{array} { l } 
{ [ E _ { 2 } ^ { \prime } , E _ { 3 } ^ { \prime } ] = 0 } \\
{ [ E _ { 2 } ^ { \prime } , E _ { 5 } ^ { \prime } ] = - 2 E _ { 6 } ^ { \prime } ] = 0 } \\
{ [ E _ { 2 } ^ { \prime } , E _ { 7 } ^ { \prime } ] = - 2 E _ { 4 } ^ { \prime } , }
\end{array} \quad \left\{\begin{array}{l}
\left.\left[E_{3}^{\prime}, E_{4}^{\prime}\right]=E_{2}^{\prime}, E_{6}^{\prime}, E_{5}^{\prime}\right]=-E_{3}^{\prime} \\
{\left[E_{3}^{\prime}, E_{6}^{\prime}\right]=E_{3}^{\prime}} \\
{\left[E_{3}^{\prime}, E_{7}^{\prime}\right]=E_{5}^{\prime}-E_{6}^{\prime},}
\end{array}\right.\right.\right.
\end{aligned}
$$

Now, we are in the position to prove the main result of this paper. Suppose that the $G$-action extends on $\widehat{\mathcal{W}}$. This implies that we also have 7 formal vector fields $E_{1}, E_{2}, E_{3}, E_{4}, E_{5}, E_{6}, E_{7}$ on $\widehat{\mathcal{W}}$ with the following Lie bracket constraints

$$
\begin{aligned}
& \left\{\begin{array} { l } 
{ [ E _ { 1 } , E _ { 2 } ] = 0 } \\
{ [ E _ { 1 } , E _ { 3 } ] = - 2 E _ { 4 } } \\
{ [ E _ { 1 } , E _ { 4 } ] = 0 } \\
{ [ E _ { 1 } , E _ { 5 } ] = 0 } \\
{ [ E _ { 1 } , E _ { 6 } ] = - 2 E _ { 1 } } \\
{ [ E _ { 1 } , E _ { 7 } ] = 0 , }
\end{array} \quad \left\{\begin{array} { l } 
{ [ E _ { 2 } , E _ { 3 } ] = 0 } \\
{ [ E _ { 2 } , E _ { 4 } ] = 0 } \\
{ [ E _ { 2 } , E _ { 5 } ] = - 2 E _ { 2 } } \\
{ [ E _ { 2 } , E _ { 6 } ] = 0 } \\
{ [ E _ { 2 } , E _ { 7 } ] = - 2 E _ { 4 } , }
\end{array} \quad \left\{\begin{array}{l}
{\left[E_{3}, E_{4}\right]=E_{2}} \\
{\left[E_{3}, E_{5}\right]=-E_{3}} \\
{\left[E_{3}, E_{6}\right]=E_{3}} \\
{\left[E_{3}, E_{7}\right]=E_{5}-E_{6},}
\end{array}\right.\right.\right.
\end{aligned}
$$

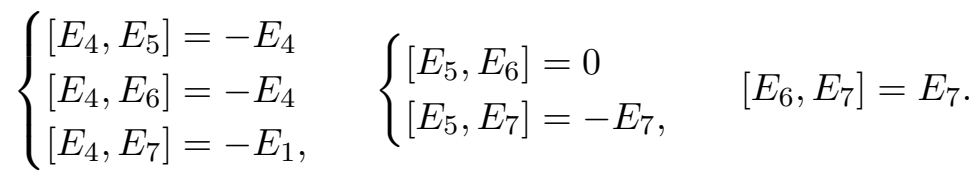

These vector fields form a Lie subalgebra, denoted by $\mathfrak{g}$, of the Lie algebra of formal vector fields on $\widehat{\mathcal{W}}$. Of course, the restriction of $E_{i}$ on the central fiber is nothing but $E_{i}^{\prime}(i=1, \ldots, 7)$. 
From the previous section, we can assume that our seven vector fields are of the form

$$
E_{i}=g_{i}(v, t) \frac{\partial}{\partial v}+\left(\alpha_{i}(v, t) y^{2}+\beta_{i}(v, t) y+\gamma_{i}(v, t)\right) \frac{\partial}{\partial y}+k_{i}(t) \frac{\partial}{\partial t},
$$

(cf. Lemma 3.1) where $A, B, C, a, b, c, e$ are formal power series in $t(i=1, \ldots, 7)$.

Theorem 4.1. The action of $G$ on $\mathbb{F}_{2}$ does not extend to the formal semi-universal deformation $\widehat{\mathcal{W}}$, where $G$ is the automorphism group of $\mathbb{F}_{2}$.

Proof. We denote by $\mathfrak{v}$ the Lie algebra of formal vector fields in one variable $t$. Let $\delta: \mathfrak{g} \rightarrow \mathfrak{v}$ be the map which sends

$$
g_{i}(v, t) \frac{\partial}{\partial v}+\left(\alpha_{i}(v, t) y^{2}+\beta_{i}(v, t) y+\gamma_{i}(v, t)\right) \frac{\partial}{\partial y}+k_{i}(t) \frac{\partial}{\partial t}
$$

to

$$
k_{i}(t) \frac{\partial}{\partial t}
$$

for $i=1, \ldots, 7$. Since, the first two components $\frac{\partial}{\partial v}$ and $\frac{\partial}{\partial y}$ contribute nothing to the component $\frac{\partial}{\partial t}$ in the Lie bracket then $\delta$ is a well-defined Lie homomorphism. Set $F_{i}:=\delta\left(E_{i}\right)=k_{i}(t) \frac{\partial}{\partial t}$ $(i=1, \ldots, 7)$. Note that the seven formal vector fields $F_{i}(i=1, \ldots, 7)$ are nothing but those induced by the $G$-action on the base $\operatorname{Specf}(\mathbb{C}[[t]])$ (cf. the last paragraph of Section 1 ). Observe also that $\mathfrak{v}$ can be equipped with a filtration $F$ given by the vanishing order at 0 and we have two well-known facts

$$
\left[F^{p} \mathfrak{v}, F^{p} \mathfrak{v}\right] \subset F^{2 p} \mathfrak{v}, \text { and }\left[F^{p} \mathfrak{v}, F^{q} \mathfrak{v}\right] \subset F^{p+q-1} \mathfrak{v},
$$

for $p, q \geq 1$. Furthermore, the vanishing order of all $k_{i}$ at 0 is at least 1 . Let $k_{i}(t)=\sum_{j=1}^{\infty} a_{j}^{i} t^{j}$ $(i=1,2,4,5)$. Using the first fact and the Lie relations induced by $\delta$ :

$$
\left\{\begin{array}{l}
{\left[F_{1}, F_{6}\right]=-2 F_{1}} \\
{\left[F_{2}, F_{5}\right]=-2 F_{2}} \\
{\left[F_{1}, F_{3}\right]=-2 F_{4},}
\end{array}\right.
$$

we obtain $a_{1}^{1}=a_{1}^{2}=a_{1}^{4}=0$. Suppose that $k_{4}(t)$ is not identically zero, then there exists $j^{*} \geq 2$ such that $a_{j^{*}}^{4}$ is nonzero. By computing explicitly the Lie relation $\left[F_{4}, F_{5}\right]=-F_{4}$ in terms of power series in $t$ and then by equalizing coefficients, we get that

$$
a_{j}^{4}\left[(j-1) a_{1}^{5}-1\right]=0,
$$

for all $j \geq 2$. Thus, $a_{1}^{5}=\frac{1}{j^{*}-1}$, which is clearly nonzero. A similar computation for the relation $\left[F_{1}, F_{5}\right]=0$ gives

$$
(j-1) a_{j}^{1} a_{1}^{5}=0,
$$

for all $j \geq 2$. Hence, all $a_{j}^{1}=0$ so that $k_{1}(t)=0$. By the relation $\left[F_{1}, F_{3}\right]=-2 F_{4}$, we deduce that $k_{4}(t)=0$, a contradiction. Therefore, $k_{4}(t)=0$. From the relations $\left[F_{3}, F_{4}\right]=F_{2}$ and $\left[F_{4}, F_{7}\right]=-F_{1}$, we obtain that $k_{2}(t)=0$ and $k_{1}(t)=0$. As a sequence, $E_{1}, E_{2}$, and $E_{4}$ do not have the component $\frac{\partial}{\partial t}$.

In addition, by the proof of Theorem 3.1 , as a scheme over $\mathbb{C}$,

$$
\mathcal{W}^{*} \cong \mathbb{C}^{*} \times \mathbb{P}^{1} \times \mathbb{P}^{1} \text {. }
$$


Then,

$$
\mathcal{W}^{*} \cong \mathbb{P}_{L}^{1} \times \mathbb{P}_{L}^{1}
$$

as a scheme over $L$, where $L:=\mathbb{C}\left[t, t^{-1}\right]$ and $\mathbb{P}_{L}^{1}$ is the 1 -projective space over $L$. Therefore, the generic fiber $\widehat{\mathcal{W}}^{*}$ of $\widehat{\mathcal{W}}$ is isomorphic to $\mathcal{W}^{*} \times_{\operatorname{Spec}\left(\mathbb{C}\left[t, t^{-1}\right]\right)} \operatorname{Spec}\left(\mathbb{C}\left[\left[t, t^{-1}\right]\right]\right)=\mathbb{P}_{K}^{1} \times \mathbb{P}_{K}^{1}$, as a scheme over $K$, where $K$ is the field of Laurent formal power series $\mathbb{C}\left[\left[t, t^{-1}\right]\right]$. Now, by restricting on the generic fiber of $\widehat{\mathcal{W}}$, we obtain that $E_{1}, E_{2}$, and $E_{4}$ are formal vector fields on $\widehat{\mathcal{W}}^{*}$, considered as a $\mathbb{C}$-scheme. However, by the first paragraph, we have proved that there is no component $\frac{\partial}{\partial t}$ in the expression of $E_{i}(i=1,2,4)$. So, if we think of $E_{1}, E_{2}$ and $E_{4}$ as vector fields with coefficients in $K$, then they are definitely vector fields on $\widehat{\mathcal{W}}^{*}$, regarded as a scheme over $K$. Note that the Lie algebra of vector fields on $\mathbb{P}_{K}^{1} \times \mathbb{P}_{K}^{1}$ is isomorphic to $\mathfrak{s l}_{2}(K) \times \mathfrak{s l}_{2}(K)$, where $\mathfrak{s l}_{2}(K)$ is the special linear group. This means that there exists a 3-dimensional abelian Lie subalgebra of $\mathfrak{s l}_{2}(K) \times \mathfrak{s l}_{2}(K)$. The image of that subalgebra under one of the two canonical projections of the product $\mathfrak{s l}_{2}(K) \times \mathfrak{s l}_{2}(K)$ provides a 2 -dimensional abelian Lie subalgebra in $\mathfrak{s l}_{2}(K)$. This is a contradiction since $\operatorname{rank}\left(\mathfrak{s l}_{2}(K)\right)$ is only 1 .

Remark 4.1. A naturally posed question is if the $G$-action extends to $\mathcal{W}_{n}$ over $\operatorname{Spec}\left(\mathbb{C}[t] /\left(t^{n+1}\right)\right)$ for small value $n$. Although the above proof does not give any clue to reply to this question, the answer is yes for $n=1$. More general, if $G$ is an algebraic group acting algebraically on a projective variety $X$ and $\pi: \mathcal{X} \rightarrow S$ is the semi-universal deformation of $X$ then the $G$-action on $X$ certainly extends up to the first infinitesimal deformation $\mathcal{X}_{1}$ over $S_{1}$. This follows easily from the semi-universality of the family $\pi: \mathcal{X} \rightarrow S$. Unfortunately, our example turns out to be the worst case. More precisely, we can even show that the $G$-action on $\mathbb{F}_{2}$ can not extend to the second infinitesimal $\mathcal{W}_{2}$ over $\operatorname{Spec}\left(\mathbb{C}[t] /\left(t^{3}\right)\right)$ by extending $E_{i}^{\prime}(i=1, \ldots, 7)$ together with their Lie bracket relations, order by order with respect to $t$. However, the computations are somewhat lengthy and complicated.

\section{REFERENCES}

1. J. Blanc, Finite subgroups of the Cremona group of the plane. The 35th Autumn School in Algebraic Geometry, Poland, September 23 - September 29 (2012).

2. R. Hartshorne, Algebraic Geometry, Springer Verlag Graduate Texts in Mathematics, Vol 52, (1977).

3. K. Kodaira, Complex Manifolds and Deformation of Complex Structures, Classics in Mathematics, English edn. Springer, Berlin, (2005).

4. D. S. Rim, Equivariant G-structure on versal deformations, Transactions of the American Mathematical Society, 257(1) (1980): 217-226.

5. M. Schlessinger, Functors of Artin rings, Transactions of the American Mathematical Society, 130(2) (1968): 208-222.

6. E. Sernesi, Deformations of algebraic schemes, Grundlehren der Mathematischen Wissenschaften, vol. 334. Springer, Berlin (2006).

7. J. J. Wavrik, Obstructions to the existence of a space of moduli, Papers in Honour of K. Kodaira, Princeton Univ. Press, (1969), 403-414.

An Khuong DOAn, IMJ-PRG, UMR 7586, Sorbonne Université, Case 247, 4 place Jussieu, 75252 Paris Cedex 05, France

E-mail address: an-khuong.doan@imj-prg.fr 